\title{
Kawasaki disease in an infant after administration of hexavalent vaccine
}

\author{
Meshal Z. Almeshary, MD, Saud A. Alanazi, MD, Khalid M. Almoosa, MD, Rolan K. Bassrawi, MD.
}

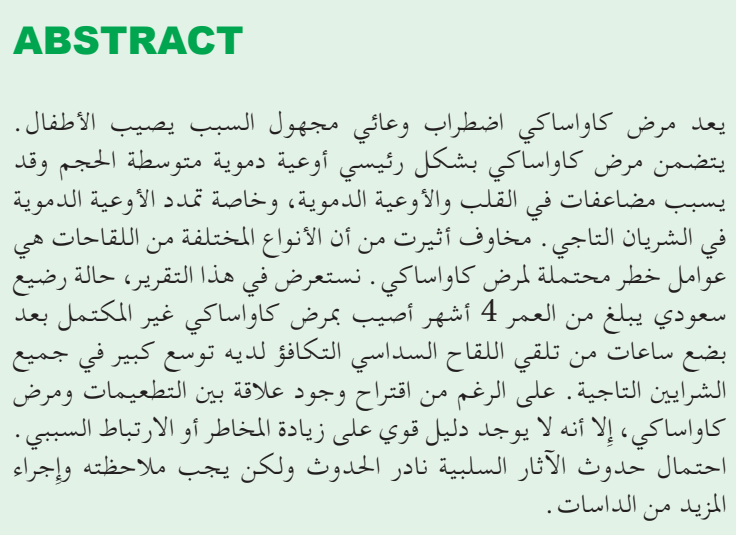

Kawasaki disease is a vascular disorder of unknown etiology that affects children. Kawasaki disease mainly involves medium-sized blood vessels and may cause cardiovascular complications, particularly coronary artery aneurysms. Concern has been raised against various types of vaccines becoming potential risk factors for Kawasaki disease. Here, we describe a case of a 4-month-old Saudi infant who presented with incomplete Kawasaki disease a few hours after receiving his hexavalent vaccine and there was a significant dilatation of all coronary arteries. Although a relationship between vaccinations and Kawasaki disease has been suggested, there is no strong evidence of an increased risk or causal association. This possibility of adverse effects is rare but should be observed and further investigated.

Keywords: hexavalent vaccine, infant, kawasaki disease

Saudi Med J 2021; Vol. 42 (7): 790-792

doi: 10.15537/smj.2021.42.7.20210061

From the Department of Pediatrics, King Saud University Medical City, Riyadh, Kingdom of Saudi Arabia.

Received 9th February 2021. Accepted 5th May 2021.

Address correspondence and reprint request to: Dr. MeshalZ. Almeshary, Department of Pediatrics, King Saud University Medical City, Riyadh, Kingdom of Saudi Arabia. E-mail: meshal4545@gmail.com ORCID ID: https://orcid.org/0000-0001-5448-9954
Kawasaki disease (KD) is an acute and self-limited Imedium-vessel vasculitis that mainly involves the coronary arteries. The pathogenesis of $\mathrm{KD}$ is still unknown. It appears that KD occurs as hyperinflammatory response in a genetically predisposed individual that is possibly triggered by infectious agents or several environmental factors. We report a case of $\mathrm{KD}$ in an infant after administration of hexavalent vaccine.

Case Report. A 4-month-old Saudi infant with an unremarkable antenatal and perinatal history.

Clinical Findings. At the age of 4 months, he presented to the emergency department with a 9-day history of fever. He was in his usual state of good health until he developed fever 8 hours post-hexavalent vaccine (DTPa-HBV-IPV/Hib). His temperature started suddenly and was measured at home as $38.9^{\circ} \mathrm{C}$ axillary by his mother with no clear focus. On examination, he appeared irritable and was febrile. His temperature upon arrival was $38.6^{\circ} \mathrm{C}$ rectally with a heart rate of 168 beats/ $\mathrm{min}$ and a respiratory rate of 38 breaths $/ \mathrm{min}$. His blood pressure was $88 / 50 \mathrm{~mm} \mathrm{Hg}$ his oxygen saturation was $98 \%$ on room air. He developed a maculopapular rash, mainly in the upper limbs. He also had non-purulent conjunctivitis and erythematous lips but no strawberry tongue. He had neither cervical lymphadenopathy nor extremity changes.

Diagnostic assessment. A laboratory investigation upon admission showed a white blood cell (WBC) count of $17.4 \times 103$ cells/ul with $52 \%$ neutrophils, hemoglobin level of $11 \mathrm{~g} / \mathrm{dL}$, mean corpuscular hemoglobin level of 27 , mean corpuscular volume of 80 , platelets of $600 / \mu \mathrm{L}$, C-reactive protein (CRP) level of $96 \mathrm{mg} / \mathrm{L}$, erythrocyte segmentation rate (ESR) of $91 \mathrm{~mm} / \mathrm{h}$, and albumin level of $19.9 \mathrm{~g} / \mathrm{L}$. The normal range for laboratories: WBC: 5000-15000 cells/ul, hemoglobin: 9.5-14 g/dl, platelets: 150-450/ul, CRP: $<5 \mathrm{mg} / \mathrm{l}$, ESR: $<15$, and albumin: 35-45 g/l. Other laboratory workups, including urine analysis and culture, liver function tests, and renal function tests, were normal. The results of laboratory tests for infectious diseases, including nasopharyngeal aspirate for virology, multiple coronavirus disease nasal 
swabs, severe acute respiratory syndrome coronavirus 2 antibodies, and blood cultures were negative. The patient was admitted with incomplete KD.

Therapeutic intervention. Upon admission, he was administered $2 \mathrm{~g} / \mathrm{kg}$ intravenous immunoglobulin (IVIG) along with a high dose of aspirin $(80 \mathrm{mg} /$ $\mathrm{kg}$ ). The fever resolved after the first IVIG dose. The aspirin dose was decreased to the antiplatelet dose (4 $\mathrm{mg} / \mathrm{kg})$. The inflammatory markers CRP $(21 \mathrm{mg} / \mathrm{L})$ and ESR $(53 \mathrm{~mm} /$ hour) improved in 4th day of admission with an increase in platelet count $(893 / \mu \mathrm{L})$. An echocardiogram (ECHO) was carried out on the second day of admission, showed significant dilatation of the coronary arteries as follows: left main coronary artery, $4.6 \mathrm{~mm}$, Z-score of 7.3 (Figure 1A); right coronary artery, $4.2 \mathrm{~mm}$ in diameter, Z-score of 6.8 ; and left anterior descending artery, $3.8 \mathrm{~mm}$, Z-score of 6.9 (Figure 1B).

Follow-up and outcomes. The ECHO was repeated twice 5 days apart after one week and showed no significant changes. The infant was discharged on a low dose of aspirin $(4 \mathrm{mg} / \mathrm{kg})$ daily. Follow-ups were scheduled with cardiology and rheumatology clinics after one month (Figure 2).

Discussion. Kawasaki disease is an acute and selflimited medium-vessel vasculitis that mainly involves the coronary arteries. The clinical manifestations of $\mathrm{KD}$ are highly variable, with no pathognomonic laboratory findings helpful for its diagnosis. ${ }^{1}$ In our case, the diagnosis of $\mathrm{KD}$ was made clinically as incomplete $\mathrm{KD}$. This was further confirmed by ECHO, which showed significant dilatation of all coronary arteries. Although the pathogenesis of $\mathrm{KD}$ remains unknown, several theories have been suggested that may explain its cause. One such theory is an environmental trigger in patients with predisposing genetic backgrounds. However, there are no strong genetic determinants, and none can be considered real markers of disease susceptibility. ${ }^{2}$ Another theory, which is more acceptable, was proposed based on the similarity of clinical features between $\mathrm{KD}$, streptococcal toxic shock syndrome, and toxic shock syndrome with evidence of superantigen-producing pathogens in all 3 diseases. These findings suggest that the immune response of $\mathrm{KD}$ does not result from a single agent but rather a final common inflammatory pathway

Disclosure. Authors have no conflict of interests, and the work was not supported or funded by any drug company.

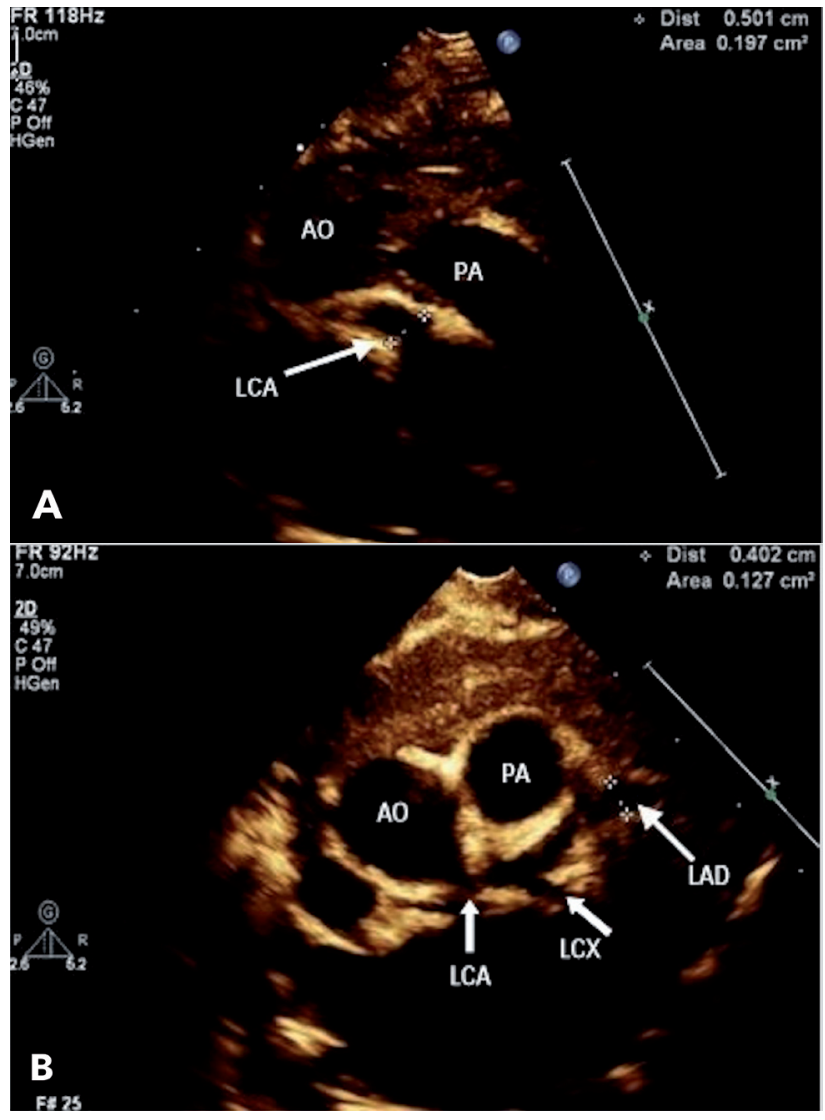

Figure 1 - Echocardiogram A) from high parasternal view showing significant dilatation of left main cornary artery (LCA), B) parasternal short axis view showing significant dilatation of left anterior descending artery (LAD). AO: aorta; PA: pulmonary artery; LCX: left circumflex artery

in genetically susceptible individuals. ${ }^{3}$ Recently, an innate immunity disorder has been proposed as a cause of $\mathrm{KD} .{ }^{4}$ Vaccine administration as a trigger for the development of $\mathrm{KD}$ remains under debate. In our case, the infant developed $\mathrm{KD}$ after receiving the hexavalent vaccine. Kawasaki disease has also been associated with other vaccines in clinical trials, observational studies, and case reports. ${ }^{5}$ In a matched case-control study in Colorado during a $\mathrm{KD}$ outbreak, 37 patients were diagnosed with $\mathrm{KD}$, but there was no evidence of an association with vaccination; the odds ratio for $\mathrm{KD}$ within 30 days following vaccination was 1.3 (95\% CI, 0.4-4.3). ${ }^{6}$ In another study, data were extracted from the Vaccine Safety Datalink to investigate the rate of $\mathrm{KD}$ in 1,721,186 children aged 0-6 years from 1996 to 2006. This study showed no increase in $\mathrm{KD}$ incidence following vaccination. ${ }^{7}$ An observational study by Hua et $\mathrm{al}^{8}$ reviewed all $\mathrm{KD}$ cases reported by the United 
States Vaccine Adverse Event Reporting System (VAERS) between 1990 and 2007. Ninety-seven cases were reported to VAERS as classic, atypical, and possible KD. Overall, the review date did not show an increased risk for all licensed vaccines. ${ }^{8}$ In an observerblinded, randomized, controlled, 5-center study, 433 participants were randomly allocated to receive a single dose of Pentacel ${ }^{\mathrm{TM}}$ or Infanrix (DTaP-IPV-Hib). Only one case of KD was reported at 3 weeks after the Infanrix vaccination. ${ }^{9}$ A review of 15 studies published between 2000 and 2016 showed that the hexavalent vaccine was generally well-tolerated, and no vaccine-related KD cases were reported. ${ }^{10}$ In this case report, we discussed a case of KD that developed following the administration of hexavalent. In this case report, we discussed a very rare adverse event of post-hexavalent vaccine.

In conclusion, we cannot hypothesize that hexavalent vaccination is a trigger for $\mathrm{KD}$ based on a single case report, and the association observed in this child may have been coincidental.

Acknowledgment. We would like to thank Editage (www.editage.com) for English language editing.

\section{References}

1. Burns JC, Glodé MP. Kawasaki syndrome. Lancet 2004; 364: 533-544.
2. Principi N, Rigante D, Esposito S. The role of infection in Kawasaki syndrome. J Infect 2013; 67: 1-10.

3. Matsubara K, Fukaya T. The role of superantigens of group A Streptococcus and Staphylococcus aureus in Kawasaki disease. Curr Opin Infect Dis 2007; 20: 298-303.

4. Hara T, Nakashima Y, Sakai Y, Nishio H, Motomura Y, Yamasaki S. Kawasaki disease: a matter of innate immunity. Clin Exp Immunol 2016; 186: 134-143.

5. Phuong LK, Bonetto C, Buttery J, Pernus YB, Chandler R, Felicetti P, et al. Kawasaki disease and immunisation: a systematic review. Vaccine 2017; 35: 1770-1779.

6. Treadwell TA, Maddox RA, Holman RC, Belay ED, Shahriari A, Anderson MS, et al. Investigation of Kawasaki syndrome risk factors in Colorado. Pediatr Infect Dis J 2002; 21: 976-978.

7. Abrams JY, Weintraub ES, Baggs JM, McCarthy NL, Schonberger LB, Lee GM, et al. Childhood vaccines and kawasaki disease, vaccine safety datalink, 1996-2006. Vaccine 2015; 33: 382-387.

8. Hua W, Izurieta HS, Slade B, Penina Haber, Rosemary Tiernan, et al. Table, supplemental digital content 1- vaccine adverse event reporting system (Vaers) case definitions of Kawasaki disease (Kd). Pediatr Infect Dis J 2009; 28: 943-947.

9. Halperin SA, Tapiero B, Law B, Diaz-Mitoma F, Duval B, Langley JM, et al. Interchangeability of two diphtheria and tetanus toxoids, acellular pertussis, inactivated poliovirus, Haemophilus influenzae type $b$ conjugate vaccines as a fourth dose in 15-20-month-old toddlers. Vaccine 2006; 24 : 4017-4023.

10. Bulik NB. Hexavalent vaccine in Europe: safety data from the randomized clinical trials. Farmacia 2018; 66: 747-757. 